PROCEEDINGS OF THE

AMERICAN MATHEMATICAL SOCIETY

Volume 140, Number 4, April 2012, Pages 1393-1396

S 0002-9939(2011)10990-0

Article electronically published on August 5, 2011

\title{
ON THE RELATIVE WEAK ASYMPTOTIC HOMOMORPHISM PROPERTY FOR TRIPLES OF GROUP VON NEUMANN ALGEBRAS
}

\author{
PAUL JOLISSAINT
}

(Communicated by Marius Junge)

\begin{abstract}
A triple of finite von Neumann algebras $B \subset N \subset M$ is said to have the relative weak asymptotic homomorphism property if there exists a net of unitaries $\left(u_{i}\right)_{i \in I} \subset U(B)$ such that

$$
\lim _{i \in I}\left\|\mathbb{E}_{B}\left(x u_{i} y\right)-\mathbb{E}_{B}\left(\mathbb{E}_{N}(x) u_{i} \mathbb{E}_{N}(y)\right)\right\|_{2}=0
$$

for all $x, y \in M$. Recently, J. Fang, M. Gao and R. Smith proved that the triple $B \subset N \subset M$ has the relative weak asymptotic homomorphism property if and only if $N$ contains the set of all $x \in M$ such that $B x \subset \sum_{i=1}^{n} x_{i} B$ for finitely many elements $x_{1}, \ldots, x_{n} \in M$. Furthermore, if $H<G$ is a pair of groups, they get a purely algebraic characterization of the weak asymptotic homomorphism property for the pair of von Neumann algebras $L(H) \subset L(G)$, but their proof requires a result which is very general and whose proof is rather long. We extend the result to the case of a triple of groups $H<K<G$, we present a direct and elementary proof of the above-mentioned characterization, and we introduce three more equivalent conditions on the triple $H<K<G$, one of them stating that the subspace of $H$-compact vectors of the quasi-regular representation of $H$ on $\ell^{2}(G / H)$ is contained in $\ell^{2}(K / H)$.
\end{abstract}

\section{INTRODUCTION}

Let $1 \in B \subset N \subset M$ be a triple of finite von Neumann algebras endowed with a fixed, normal, finite, faithful and normalized trace $\tau$. Then $\mathbb{E}_{N}\left(\right.$ resp. $\left.\mathbb{E}_{B}\right)$ denotes the $\tau$-preserving conditional expectation from $M$ onto $N$ (resp. $B$ ); we also set $M \ominus N=\left\{x \in M: \mathbb{E}_{N}(x)=0\right\}$.

Following [3], we say that the triple $B \subset N \subset M$ has the relative weak asymptotic homomorphism property if there exists a net of unitaries $\left(u_{i}\right)_{i \in I} \subset U(B)$ such that, for all $x, y \in M$,

$$
\lim _{i \in I}\left\|\mathbb{E}_{B}\left(x u_{i} y\right)-\mathbb{E}_{B}\left(\mathbb{E}_{N}(x) u_{i} \mathbb{E}_{N}(y)\right)\right\|_{2}=0 .
$$

Using the identity

$$
\mathbb{E}_{B}(x u y)-\mathbb{E}_{B}\left(\mathbb{E}_{N}(x) u \mathbb{E}_{N}(y)\right)=\mathbb{E}_{B}\left(\left[x-\mathbb{E}_{N}(x)\right] u\left[y-\mathbb{E}_{N}(y)\right]\right)
$$

Received by the editors November 8, 2010 and, in revised form, November 18, 2010 and January $5,2011$.

2010 Mathematics Subject Classification. Primary 46L10; Secondary 22D25.

Key words and phrases. von Neumann algebra, one-sided quasi-normalizer, discrete group, quasi-regular representation, asymptotic homomorphism. 
which holds for every $u \in U(B)$ and all $x, y \in M$, it is readily seen that the triple $B \subset N \subset M$ has the relative weak asymptotic homomorphism property if and only if one can find a net $\left(u_{i}\right)_{i \in I} \subset U(B)$ such that

$$
\lim _{i \in I}\left\|\mathbb{E}_{B}\left(x u_{i} y\right)\right\|_{2}=0
$$

for all $x, y \in M \ominus N$.

The one-sided quasi-normalizer of $B$ in $M$ is the set of elements $x \in M$ for which there exist finitely many elements $x_{1}, \ldots, x_{n} \in M$ such that $B x \subset \sum_{i=1}^{n} x_{i} B$. It is denoted by $q \mathcal{N}_{M}^{(1)}(B)$.

Inspired by [2, the authors of 3 prove in Theorem 3.1 that the triple $B \subset$ $N \subset M$ has the relative weak asymptotic homomorphism property if and only if $q \mathcal{N}_{M}^{(1)}(B) \subset N$. Furthermore, they also study the case of group algebras that we recall now.

Let $G$ be a discrete group and let $H$ be a subgroup of $G$. Then there is a natural analogue of the one-sided quasi-normalizer for such a pair of groups: we denote by $q \mathcal{N}_{G}^{(1)}(H)$ the set of elements $g \in G$ for which there exist finitely many elements $g_{1}, \ldots, g_{n} \in G$ such that $H g \subset \bigcup_{i=1}^{n} g_{i} H$.

Thus, if $H<K<G$ is a triple of groups and if $B=L(H) \subset N=L(K) \subset$ $M=L(G)$ denotes the triple of von Neumann algebras associated to $H<K<G$, then it is reasonable to ask whether $B \subset N \subset M$ has the relative weak asymptotic homomorphism property if and only if $q \mathcal{N}_{G}^{(1)}(H) \subset K$. Corollary 5.4 in [3] states that this is indeed true when $K=H$, but the proof presented there relies heavily on the main theorem of the article. It is thus natural to look for a more direct and elementary proof of the above-mentioned result, and the aim of the present paper is to provide such a proof and to add three more equivalent conditions.

\section{THE MAIN RESULT}

Before stating our result, let us fix some additional notation. For each element $g \in G$ we denote by $\lambda_{g}$ the unitary operator acting by left translation on $\ell^{2}(G)$, i.e. $\left(\lambda_{g} \xi\right)\left(g^{\prime}\right)=\xi\left(g^{-1} g^{\prime}\right)$ for every $\xi \in \ell^{2}(G)$ and every $g^{\prime} \in G$. We denote also by $L_{f}(G)$ the subalgebra of all elements of $L(G)$ with finite support; i.e., $L_{f}(G)$ is the linear span of $\lambda(G)$ in $B\left(\ell^{2}(G)\right)$.

We fix a triple of groups $H<K<G$ for the rest of the article.

Let $\pi$ denote the quasi-regular representation of $G$ on $\ell^{2}(G / H)$; we denote by $[g]$ the equivalence class $[g]=g H$, so that $\pi(g) \xi\left(\left[g^{\prime}\right]\right)=\xi\left(\left[g^{-1} g^{\prime}\right]\right)$ for all $g, g^{\prime} \in G$ and $\xi \in \ell^{2}(G / H)$. Following [1], we say that a vector $\xi \in \ell^{2}(G / H)$ is $H$-compact if the norm closure of its $H$-orbit $\{\pi(h) \xi: h \in H\}$ is a compact subset of $\ell^{2}(G / H)$. The set of all $H$-compact vectors is a closed subspace of $\ell^{2}(G / H)$ that we denote by $\ell^{2}(G / H)_{c, H}$. We also set $\ell^{2}(G / H)^{H}=\left\{\xi \in \ell^{2}(G / H): \pi(h) \xi=\xi \forall h \in H\right\}$, which is the subspace of all $H$-invariant vectors of $\ell^{2}(G / H)$. It is contained in $\ell^{2}(G / H)_{c, H}$.

Theorem 2.1. Let $H<K<G$ and $B=L(H) \subset N=L(K) \subset M=L(G)$ be as above. Then the following conditions are equivalent:

(1) There exists a net $\left(h_{i}\right)_{i \in I} \subset H$ such that, for all $x, y \in M \ominus N$, one has

$$
\lim _{i \in I}\left\|\mathbb{E}_{B}\left(x \lambda_{h_{i}} y\right)\right\|_{2}=0
$$


i.e., the net of unitaries in the relative weak asymptotic homomorphism property may be chosen in the subgroup $\lambda(H)$ of $U(B)$.

(2) The triple $B \subset N \subset M$ has the relative weak asymptotic homomorphism property.

(3) If $g \in G$ and $F \subset G$ finite are such that $H g \subset F H$, then $g \in K$, i.e. $q \mathcal{N}_{G}^{(1)}(H) \subset K$.

(4) The subspace of $H$-compact vectors $\ell^{2}(G / H)_{c, H}$ is contained in $\ell^{2}(K / H)$.

(5) The subspace of $H$-fixed vectors $\ell^{2}(G / H)^{H}$ is contained in $\ell^{2}(K / H)$.

(6) For every nonempty finite set $F \subset G \backslash K$, there exists $h \in H$ such that

$$
F h F \cap H=\emptyset .
$$

Proof. (1) $\Rightarrow(2)$ is obvious.

$(2) \Rightarrow(3)$. Observe that condition (3) is equivalent to the following statement (since, if $g \notin K$, then $H g H \cap K=\emptyset$ ):

For every $g \in G \backslash K$, and for every nonempty finite set $F \subset G \backslash K$, there exists $h \in H$ such that Fhg $\cap H=\emptyset$.

Thus, let us assume that condition (3) does not hold. There exists $g \in G \backslash K$ and a nonempty finite set $F \subset G \backslash K$ such that $F h g \cap H \neq \emptyset$ for every $h \in H$. Then let $u \in U(B)$. One has:

$$
\begin{aligned}
\sum_{g^{\prime} \in F}\left\|\mathbb{E}_{B}\left(\lambda_{g^{\prime}} u \lambda_{g}\right)\right\|_{2}^{2} & =\sum_{g^{\prime} \in F}\left(\sum_{h \in H, g^{\prime} h g \in H}|u(h)|^{2}\right) \\
& =\sum_{h \in H}\left(\sum_{g^{\prime} \in F, g^{\prime} h g \in H}|u(h)|^{2}\right) \\
& \geq \sum_{h \in H}|u(h)|^{2}=\|u\|_{2}^{2}=1
\end{aligned}
$$

since, for every $h \in H$, one can find $g^{\prime}(h) \in F$ such that $g^{\prime}(h) h g \in H$. Hence there cannot exist a net $\left(u_{i}\right)_{i \in I} \subset U(B)$ as above, and the triple $B \subset N \subset M$ does not have the relative weak asymptotic homomorphism property.

$(3) \Rightarrow(4)$. We choose a set of representatives $T \ni e$ of left classes so that $G=\bigsqcup_{t \in T} t H$, and let $\xi \neq 0$ be an $H$-compact vector.

Let $s \in T$ be such that $\epsilon:=|\xi([s])|>0$. There exist then finitely many vectors $\xi_{1}, \ldots, \xi_{n} \in \ell^{2}(G / H)$ such that, for every $h \in H$, there exists $1 \leq j \leq n$ such that $\left\|\pi(h) \xi-\xi_{j}\right\| \leq \epsilon / 2$. Set

$$
F=\bigcup_{j=1}^{n}\left\{t \in T:\left|\xi_{j}([t])\right| \geq \epsilon / 2\right\}
$$

which is a finite set. Then we claim that $H s \subset F H$. Indeed, if $h \in H$, let $t \in T$ be such that $[h s]=[t]$, and let $j$ be such that $\left\|\pi(h) \xi-\xi_{j}\right\| \leq \epsilon / 2$. Then

$$
\epsilon-\left|\xi_{j}([t])\right|=|\xi([s])|-\left|\xi_{j}([t])\right| \leq\left|\xi([s])-\xi_{j}([h s])\right| \leq\left\|\pi(h) \xi-\xi_{j}\right\| \leq \epsilon / 2 ;
$$

hence $\epsilon / 2 \leq\left|\xi_{j}([t])\right|$ and $t \in F$. Thus $H s \subset F H$, and condition (3) implies that $s \in K$. This proves that $\xi \in \ell^{2}(K / H)$.

$(4) \Rightarrow(5)$ is obvious.

$(5) \Rightarrow(6)$. Let us assume that the triple $H<K<G$ satisfies condition (5) but not (6). Then there exists a finite set $F=F^{-1} \subset G \backslash K$ such that $F h F \cap H \neq \emptyset$ 
for every $h \in H$. Set

$$
\xi=\sum_{g \in F} \delta_{[g]} .
$$

Then $\xi \perp \ell^{2}(K / H)$, and one has for every $h \in H$ :

$$
\langle\pi(h) \xi, \xi\rangle=\sum_{g, g^{\prime} \in F}\left\langle\delta_{[h g]}, \delta_{\left[g^{\prime}\right]}\right\rangle \geq 1
$$

since the condition on $F$ implies that for every $h \in H$, there exist $g, g^{\prime} \in F$ such that $h g H=g^{\prime} H$. Let $C$ be the closed convex hull of $\{\pi(h) \xi: h \in H\}$. Then it is easy to see that $\langle\zeta, \xi\rangle \geq 1$ for every $\zeta \in C$. Let $\eta \in C$ be the vector with minimal norm. By its uniqueness, it is $H$-invariant and nonzero by the above observation. Thus, $\eta$ is supported in $K / H$ and orthogonal to $\ell^{2}(K / H)$ since $\xi$ is. This is the expected contradiction.

$(6) \Rightarrow(1)$. Let $I=\{F \subset G \backslash K: F \neq \emptyset$, finite $\}$ be the directed set of all nonempty finite subsets of $G \backslash K$. Condition (6) states that, for every $F \in I$, there exists $h_{F} \in H$ such that $F h_{F} F \cap H=\emptyset$. Let $x$ and $y$ in $L_{f}(G)$ satisfy $\mathbb{E}_{N}(x)=\mathbb{E}_{N}(y)=0$. Then let $F_{0} \in I$ be chosen so that the supports of $x$ and $y$ are contained in $F_{0}$. Then $x \lambda_{h_{F}} y=\sum_{g, g^{\prime} \in F_{0}} x(g) y\left(g^{\prime}\right) \lambda_{g h_{F} g^{\prime}}$ for every $F \supset F_{0}$; thus $\mathbb{E}_{B}\left(x \lambda_{h_{F}} y\right)=0$ for every $F \supset F_{0}$. This proves that the triple $B \subset N \subset M$ satisfies condition (1) by the density of $L_{f}(G)$ in $L(G)$.

Remark 2.2. In the case of a pair of groups $H<G$, which corresponds to $H=K$, condition (4) means that all $H$-invariant vectors in $\ell^{2}(G / H)$ are multiples of $\delta_{[e]}$, and this means that the unitary representation $\rho$ of $H$ on the subspace $\ell^{2}(G / H) \ominus$ $\mathbb{C} \delta_{[e]}$ is ergodic in the sense of [1].

\section{REFERENCES}

1. V. Bergelson and J. Rosenblatt, Mixing actions of groups, Illinois J. Math. 32 (1988), 65-80. MR921351 (89g:28029)

2. I. Chifan, On the normalizing algebra of a MASA in a $I I_{1}$ factor, arXiv:math.OA/0606225, 2006.

3. J. Fang, M. Gao and R. R. Smith, The relative weak asymptotic homomorphism property for inclusions of finite von Neumann algebras, arXiv:math.OA/1005.3049 v1, 2010.

Université de Neuchâtel, Institut de Mathémathiques, Emile-Argand 11, 2000 NeuCHÂTEL, SWITZERLAND

E-mail address: paul.jolissaint@unine.ch 